\title{
NUMERICAL ANALYSIS OF THE PERFORMANCES OF THE BONDED COMPOSITE PATCH FOR REDUCING STRESS CONCENTRATION AND REPAIRING CRACKS AT NOTCH
}

\author{
B.Bachir Bouiadjra, B.Serier, D.Ouinas and B.Boutabout \\ LECM, Department of Mechanical Engineering, University of Sidi Bel Abbes, \\ BP 89, Cité Ben M'hidi, Sidi Bel Abbes, 22000, Algeria \\ e-mail : bachirbou@yahoo.fr
}

\begin{abstract}
In this paper, the finite element analysis is applied to study the performance of the bonded composite reinforcement or repair for reducing stress concentration at a semi-circular lateral notch and repairing cracks emanating from this kind of notch. The effects of the adhesive properties on the variation of the stress intensity factor at the crack tip were highlighted. The obtained results show that the stress concentration factor at the notch tip is reduced about $30 \%$ and the maximal reduction of the stress intensity factor is about $80 \%$. The adhesive properties must be optimised in order to increase the performance of the patch repair or reinforcement.
\end{abstract}

\section{Intoduction}

Externally bonded composites patches have proved to be an effective method of repairing cracks or defects in aircraft structures. This technology was pioneered by the aeronautical and maritime research laboratory of the Royal Australian air force under the direction of Alan Backer $[1,4]$. The bonded composite repair offers many advantages over a mechanically fastened doubler which include improved fatigue behaviour, reduced corrosion and easy performance to complex aerodynamic contours. It is known that the notches are the main causes of crack initiation. This is why the use of the bonded composite repair can play a significant role in the improvement of fatigue life of the notched structures. This improvement intervenes on the one hand, in the fatigue life initiation by the reduction of the stress concentration at the notch tip, and on the other hand in the fatigue life propagation by the reduction of the stress intensity of stresses at the crack tip. With the increase in computational power, the use of the numerical method, especially the finite elements method, contributed considerably to the comprehension of the mechanical behaviour of defects under patch repair. Among the authors whom used the finite element method for the study of the crack patching, we can quote : Ting et al [7], Callinan et al [8), Jones and Chiu [9], Turaga and Ripudiman [10], Chung and Yang [11], Schubbe and Mall [12] and Bachir et al [13]. The most of the previous works were interested at the cases of central patched cracks. The scope of this study covers studying the reduction of: the notch effect and the stress intensity factor at cracks emanating from notch by the bonded composite reinforcements or repairs using the finite element method. Various Authors [14,15] showed that in practice the adhesive properties plays an important role on the performance of the bonded composite patches s. For that the effects of the adhesive shear modulus and adhesive thickness on the stress intensity factor of repaired notch cracks were examined. 


\section{Geometrical and finite element models}

Consider a thin elastic aluminium plate with a semi circular lateral notch having the following dimension: height $\mathrm{Hp}=150 \mathrm{~mm}$, width $\mathrm{W}_{\mathrm{p}}=75 \mathrm{~mm}$, thickness $\mathrm{e}_{\mathrm{p}}=1.3 \mathrm{~mm}$ and notch radius $\rho=12.5 \mathrm{~mm}$. The elastic properties of the material are: Young's modulus $\mathrm{E}_{\mathrm{p}}=72$ $000 \mathrm{MPa}$, Poisson's ratio $v_{\mathrm{p}}=0.33$. The plate is subjected to uniaxial tensile load giving a remote stress state of $\sigma_{0}=70 \mathrm{MPa}$. A crack of length a emanating from the notch tip and parallel to the axis loading is supposed to exist. A Boron/epoxy patch is bonded to the plate as shown in Fig. 1. The dimensions of the patch are: Width $\mathrm{W}_{\mathrm{r}}=75 \mathrm{~mm}$ and Thickness $\mathrm{e}_{\mathrm{r}}=1.3$ $\mathrm{mm}$. The material properties of the patch are: Young's modulus $\mathrm{E}_{\mathrm{r} 1}=172369 \mathrm{MPa}, \mathrm{E}_{\mathrm{r} 2}=10342$ $\mathrm{MPa}$, Shear modulus $\mathrm{G}_{\mathrm{r}}=4826 \mathrm{MPa}$ and Poisson's ratio $v_{\mathrm{r}}=0.3$. The adhesive properties are : Shear modulus $\mathrm{G}_{\mathrm{a}}$ and thickness $\mathrm{e}_{\mathrm{a}}$. The plane stress conditions are assumed. Fig. 1 shows the geometrical model of the structures.

The total structure (plate and patch) is meshed using standard eight noded serendipity elements with quadratic shape functions. These elements perform well for elastic analysis and have the advantage that the stress singularity at the crack tip can be incorporated in the solution by moving the eight nodes to the quarter-point locations. Fig. 2 shows typical mesh model of the plate, the patch and near the crack tip.

The stress intensity factors, which govern the fracture process in LEFM context, are calculated using modified crack closure techniques.

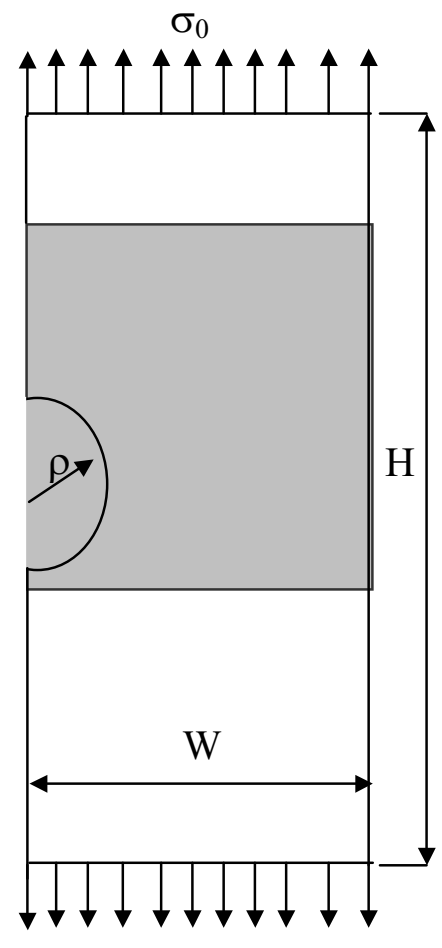

$\sigma_{0}$

FIGURE 1. Geometrical model. 


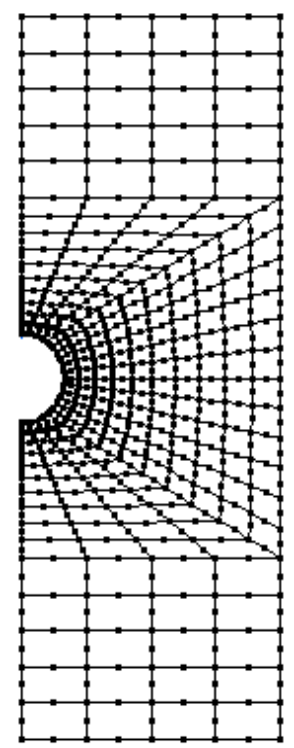

a)

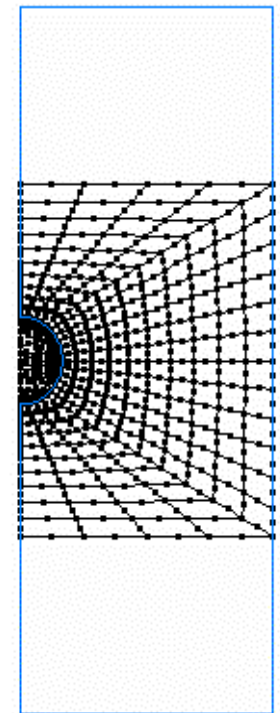

b)

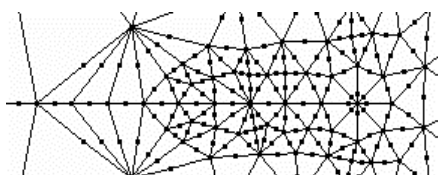

c)

FIGURE 2. Typical FE mesh : a) of the plate, b) of the patch and c) near the crack tip

\section{Analysis and Results}

\subsection{Reduction of the stress concentration at the notch tip}

Fig. 3 shows the contour of the normal stress levels $\sigma_{y}$ in the plate with the presence of the patch. The patch thickness is taken $\mathrm{e}_{\mathrm{r}}=1.3 \mathrm{~mm}$ and the adhesive properties are chosen such as $\mathrm{G}_{\mathrm{a}}=413 \mathrm{MPa}$ and $\mathrm{e}_{\mathrm{a}}=0.13 \mathrm{~mm}$. It can be seen that that the maximal stress located at the notch tip is reduced by the presence of the patch. Indeed, the stress concentration factor ( defined as $\left.\mathrm{K}_{\mathrm{t}}=\sigma_{\max } / \sigma_{0}\right)$ is limited to 2.78 by the presence of the reinforcement whereas, for the unpatched structure the value of the stress concentration factor is included between 3 and 3.5 for the semi-circular notches [20]. This reduction is due to the fact that there is a transfer of stresses through the adhesive towards the patch.

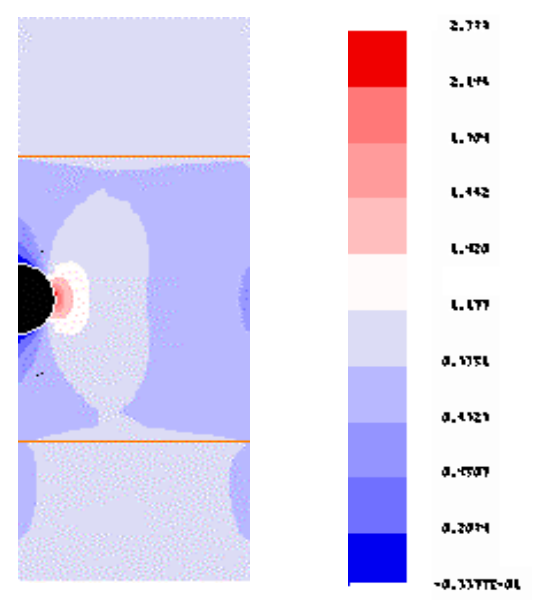

FIGURE 3. Contour of the normal stress levels in the plate. 
In Fig. 4 are presented the distribution of the normal stresses along the ligament of the plate with and without the presence of the patch for the same properties of the composite patch and the adhesive. It is shown that the maximum reduction of the stresses is about $30 \%$ but the rate of the reduction decreases progressively while moving away the notch tip. It means that the proportion of the transferred stresses towards the patch is more important since the intensity of the stresses is higher in the plate. The effect of the patch is more sensitive for levels of high stresses.

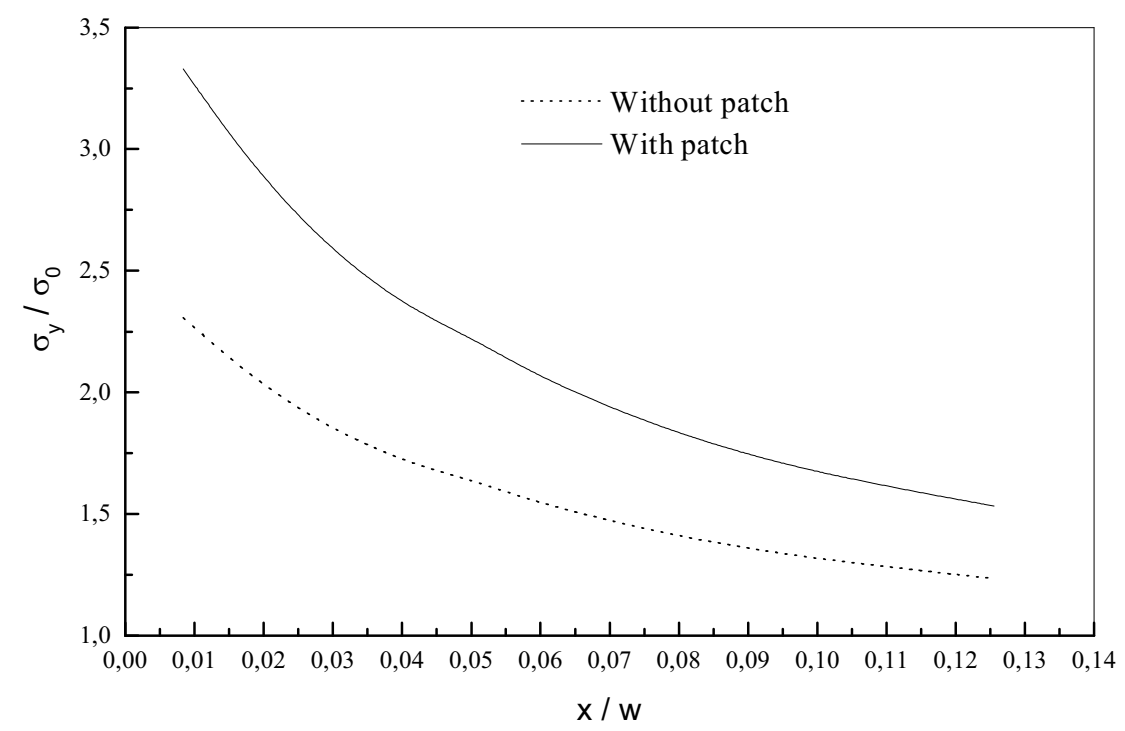

FIGURE 4. Distribution of the normal stresses along the ligament of the plate.

Fig. 5 illustrates the contour of the normal stresses $\sigma_{y}$ in the patch. The higher stresses are located at the area covering the notch, which confirm the proportionality between the stresses transmitted to the patch and that distributed on the plate without the patch. These results are confirmed in Fig. 6 where are presented the distribution of the normal stresses along the transversal axis of symmetry of the patch (axis located at the top of the ligament of the plate). The maximum stress at the patch is about 33\% of the applied stress and it is located at the free edge of the patch with the top of the notch. The stress in the patch decreases progressively and becomes zero when the stress in the plate is equal to the applied stress.

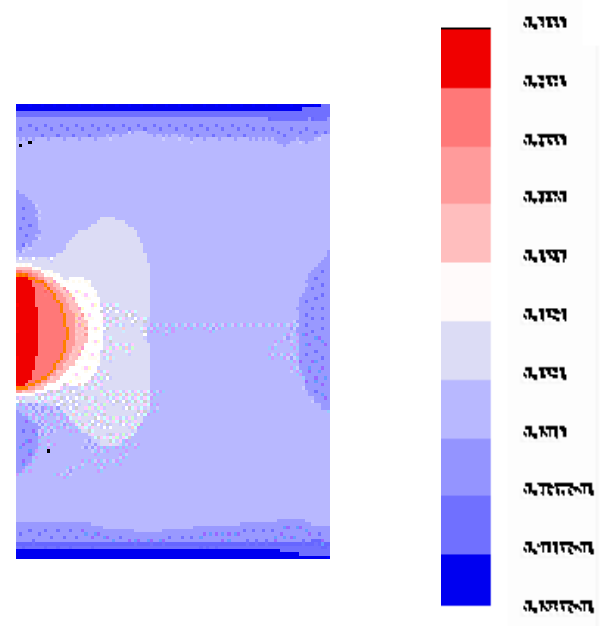

FIGURE 5. Contour of the normal stresses levels in the patch. 


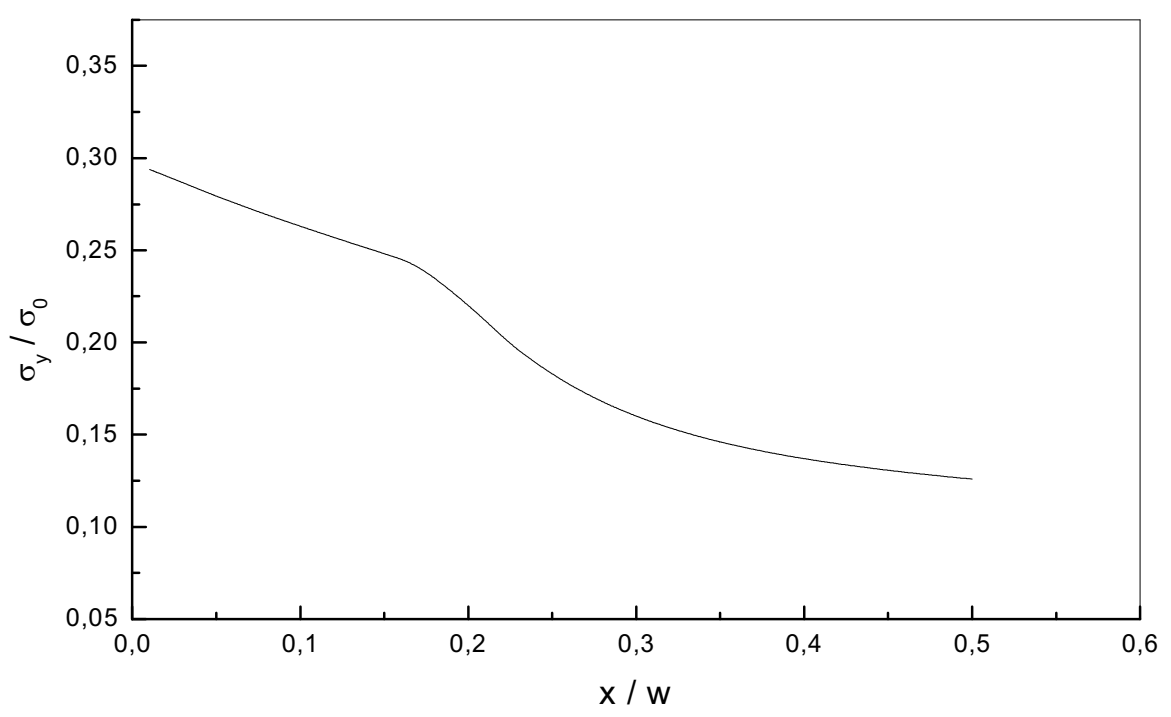

FIGURE 6. Distribution of the normal stresses along the axis of symmetry of the patch.

\subsection{Reduction of the stress intensity factor at the notch crack}

\subsubsection{Comparison between patched and unpatched crack}

Fig. 7, shows the variation of the stress intensity factor variation $\left(\mathrm{K}_{\mathrm{I}}\right)$ with respect to the crack length for patched and unpatched crack. It is shown that the patch repair highly decreases the stress intensity factor. This is because the patch carries the loads as the crack growth. The maximum reduction of $\mathrm{K}_{\mathrm{I}}$ amounts to $80 \%$. Jones and Chiu [9] give a value of $60 \%$ for a central cracks. It means the rate of reduction of stress intensity factor is more important for notch cracks. This is because the stress intensity at notch crack is higher than that at central crack. It can be seen in Fig. 7 that the stress intensity factor, for patched crack, exhibits an asymptotic behaviour as the crack length increases. Several investigators among them, those of the Australian team [8,9] announced this tendency

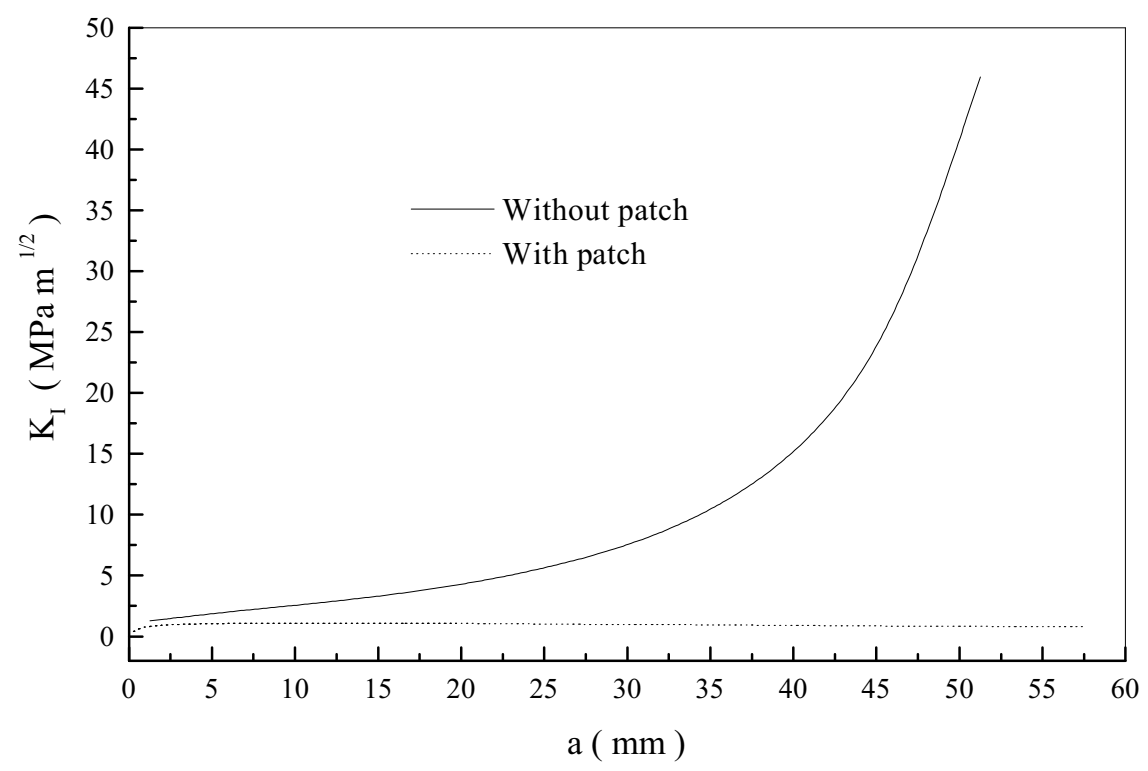

FIGURE 7. Comparison of the SIF between patched and unpatched cracks. 
By examining the shapes of the curves in Figs. 8 and 9, one can see that, according to the crack length, the stress intensity factor follows an ascending curve for small cracks , reaches a maximum then decreases and stabilises towards an asymptotic value. This behaviour is different to the case of the repaired central cracks whose stress intensity factor increases and stabilises towards an asymptotic value. This difference is explained by the fact why the stress intensity factor for short cracks emanating from notch depends on the stress concentration factor of the notch. The absorption of the stresses by the patch is less significant for short cracks emanating from notch

\subsection{Effect of the adhesive shear modulus}

It is known that the adhesives of better qualities are characterised by weak shear modulus, which make it possible to attenuate the stresses transmitted to the adhesive. In the case of repaired cracks, the objective is to transmit the maximum of the stresses to the adhesive and consequently to the patch in order to reduce them at the crack tip. Theoretically, it is thus preferable to use adhesives with high shear modulus (adhesive of bad qualities) for repairing cracks or defects. In Fig. 8, the plot of the variation of the stress intensity factor according to the crack length for various values of shear modulus of the adhesive confirms what was advanced previously. Indeed, the stress intensity factor decreases as the shear modulus of the adhesive increases, but the decrement of $K_{I}$ according to $G_{a}$ tends to be cancelled as $G_{a}$ increases indefinitely as shown in Fig. 8. Actually, an increase of the adhesive shear modulus reduces the adhesive strength, which can generate the adhesion failure.. Consequently, the choice of the adhesive (characterised by its shear modulus) for repairing cracks must be optimised in order to allow the transmission of the stresses towards the patch and to avoid the adhesive failure due to the increase of the stresses in the adhesive layer.

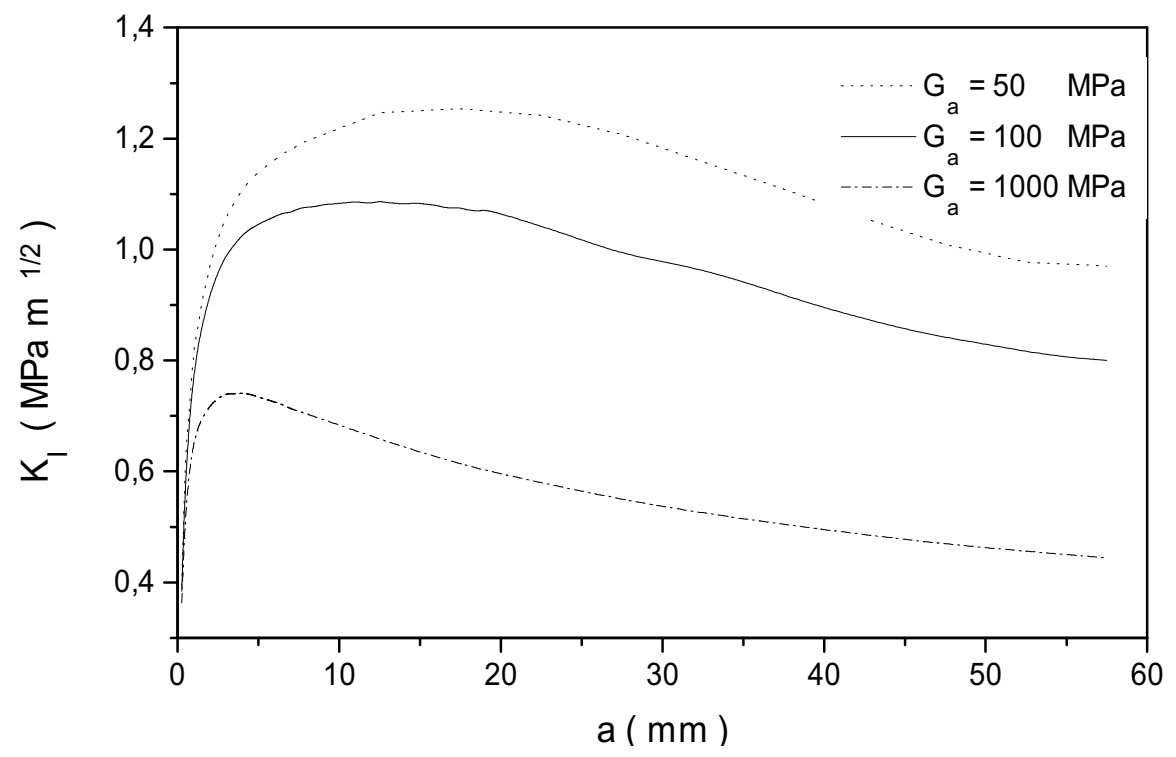

FIGURE 8. Effect of The adhesive shear modulus on the variation of the SIF.

\subsubsection{Effect of the adhesive thickness}

Fig. 9 shows the variation of the stress intensity factors as a function of the crack length for various values of the adhesive thickness $\left(\mathrm{e}_{\mathrm{a}}\right)$. It can be seen that a reduction in the adhesive thickness decreases the stress intensity factor, it means that lower adhesive thickness is 
desirable for repairing crack. This effect was highlighted by Turaga and Ripudaman [10]. However, and by analogy with what was advanced in the study of the effect of the adhesive shear modulus, It proves that the choice of the adhesive thickness must be also optimised. The high thickness reinforces adhesion but attenuates the transfer of the loads towards the patch, which reduces the beneficial effects of the patch. On the other hand, lower thickness supports the transfer of the load towards the patch but increases the risk of the adhesive failure.

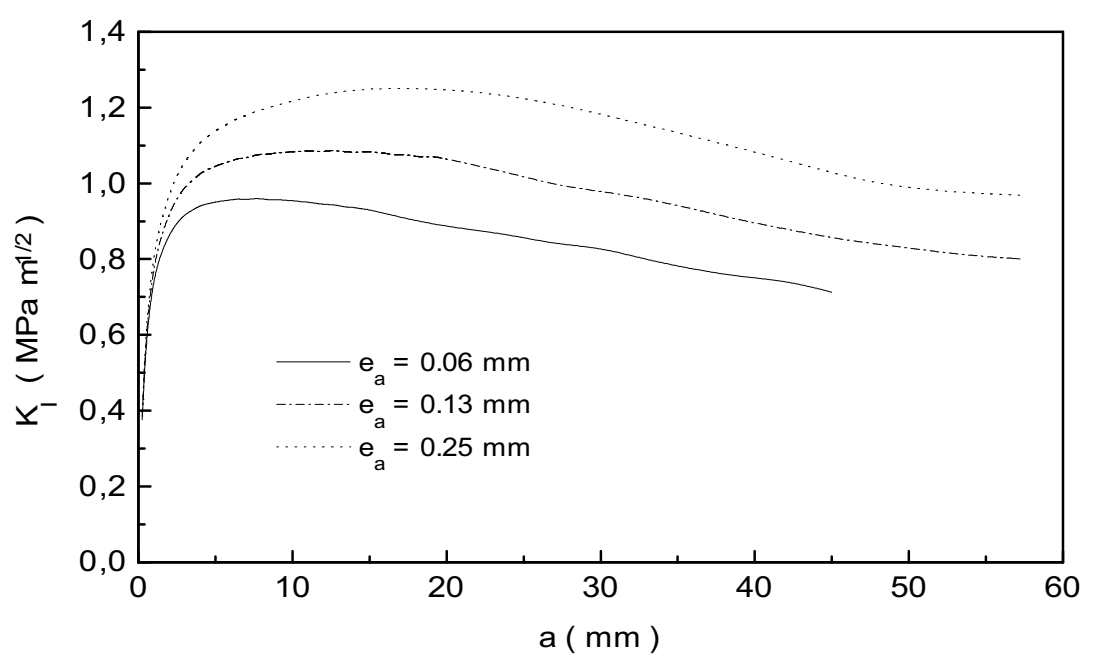

FIGURE 9. Effect of The adhesive thickness on the variation of the SIF.

\subsubsection{Effect of the patch thickness}

This effect is illustrated in Fig. 10 by the plot of the SIF variation according to the crack length for various patch thicknesses. It can be seen that the increase of the patch thickness reduces the stress intensity factor at the crack tip in a proportional way. Indeed, an increase of about $50 \%$ of the patch thickness reduces the stress intensity factor by the same order. That can able us to confirm that the choice of thicker patches makes it possible to increase their performances. For a better distribution of the stresses, it is preferable to use a multiple layers of bonded composite patches for repairing cracks.

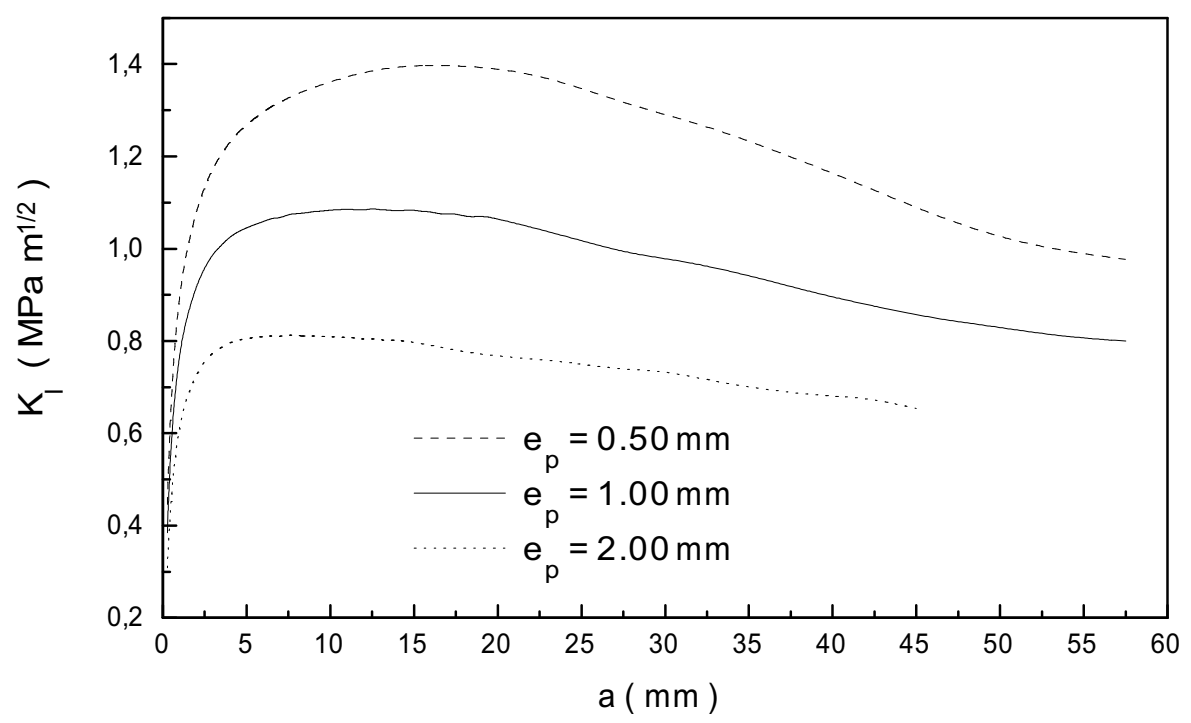

FIGURE 10. Effect of the patch thickness on the variation of the SIF 


\section{Conclusions}

The study has been made in aim to determine the performance of the bonded composite patch for reducing stress concentration and repairing crack at notch. The obtained results allow us to deduce the following conclusions:

- The reduction of the stress concentration factor by the bonded composite reinforcement is about $30 \%$, which improve the fatigue life initiation.

- The absorption of the stress by the patch is important when the stress in the reinforced plate increase.

The stress intensity factor is highly reduced by the presence of the patch.

For notch cracks, the stress intensity factor reaches a maximum value then exhibits an asymptotic behaviour as the crack length increases.

The choice of the adhesive properties for repairing crack, with the bonded composite patch, must be optimised.

The choice of the patch thickness is one of the best means for increasing the performance of the patch repair

\section{References}

1. Baker, A.A. and Chester R.J., Proceeding of the International Conference on Advanced composite Materials, 1993, 45-9.

2. Baker, A.A., In Proceeding of the Eleventh International of composite Materials (ICCM11). Gold Coast, Australia. 1997.

3. Baker, A.A., Callinan R.J., Davis M.J., Jones R. and Williams J.G., Theoritical and Applied Fracture Mechanics, vol. 2,1-16, 1984.

4. Baker, A.A., Composite Structures, vol. 2, 153-81,1984.

5. Ting,T., Jones R., Chiu W.K., Marshall I.H. and Greer J.M, Composite Structures, vol, 47,737-743, 1999.

6. Callinan, R.J., Sanderson S. and Keeley D., DSTO, Melbourne. DSTO-TN-0067, 1997

7. Jones., R. and Chiu W.K., Composite Structures, vol. 44, 17-29, 1999.

8. Turaga., V.R.S, and Ripudaman S., Engineering Fracture Mechanics, vol. 62, 267-289, 1999.

9. Chung., K.H, and Yang W.H., Composite Structures, vol. 55, 269-276, 2002.

10. Schubbe., J.J. and Mall S., Composite Structures, vol. 45, 185-193, 1999.

11. Bachir Bouiadjra B., Belhouari M. and Serier, B., Composite Structures, vol. 56, 401406, 2002.

12. Ratwani, M.M., AIAA Journal, vol. 17, 988-994, 1979.

13. Denney, J.J, and Mall S., Engineering Fracture Mechanics, vol. 57, 5, 507-525, 1997.

14. Swenson, D and James M., FRANC2D/L, Version 1.4 User's guide, 1998.

15. Kujawski, D., Fat . fract. Eng. Mat. Struct., vol. 14, 953-965. 1991. 